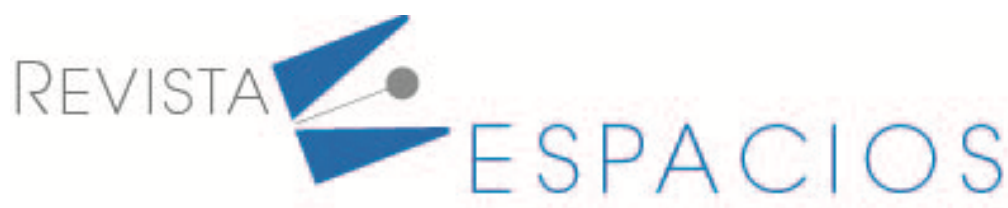

Vol. 42 (10) $2021 \cdot$ Art. 5

\title{
Gobernanza del agua y creación de valor social en el Acueducto y Alcantarillado de Popayán SA-ESP (Colombia)
}

\section{Water governance and social value creation in Acueducto y Alcantarillado de Popayan SA- ESP}

FAJARDO, Juan P. ${ }^{1}$

ROMERO, Celene M. ${ }^{2}$

Resumen

Esta investigación utilizó una encuesta a los usuarios y entrevistas y Focus Group a directivos de la empresa de Acueducto y Alcantarillado de Popayán SA-ESP, con el objetivo de diseñar una propuesta con estrategias integradoras de la gobernanza del agua y creación de valor social, donde se halla que no existen procesos de gobernanza del agua y que hay necesidad de incorporar elementos del contexto local a un modelo multinivel de gobernanza propuesto por la OCDE, que requiere la participación de todos, para generar valor social.

Palabras clave: gobernanza, agua, valor social.

\begin{abstract}
This descriptive research used a survey applied to users, an interview and a Focus Group to managers of the Aqueduct and Sewerage company of Popayan SA-ESP., with the aim of designing a proposal with integrative strategies for water governance and the creation of social value, where it is found that there are no water governance processes; and that there is a need to incorporate elements of the local context into a multilevel model of governance proposed by the OECD, which requires the participation of all, to generate social value.

key words: governance, water, social value.
\end{abstract}

\section{Introducción}

Guppy \& Anderson (2017a) explican que el agua, como fundamento de la vida, es también elemento crucial para el desarrollo sostenible. Esto autores sostienen que:

Hasta el 40\% de la población mundial vivirá en zonas con fuerte escasez de agua hacia 2035 y la capacidad de los ecosistemas para proporcionar agua dulce estará muy comprometida. El 60\% del agua dulce proviene de cuencas hidrográficas que cruzan fronteras nacionales, de manera que serán necesarios acuerdos internacionales sobre el agua para lidiar con un entorno incierto y las condiciones del cambio climático, los cambios demográficos que llevarán la población mundial a 9700 millones de personas para

\footnotetext{
${ }^{1}$ Universidad Cooperativa de Colombia. Colombia. juanpafjpf@hotmail.com

2 Universidad Cooperativa de Colombia. Colombia. celene.romeroh@campusucc.edu.co
} 
2050, doblándose el número de personas que viven en las ciudades. Una gestión efectiva del agua será necesaria para enfrentar el desperdicio del agua en los sistemas actuales, que se estima en un 30\%. (p.1).

Al mismo tiempo, Guppy \& Anderson (2017 b) expresan que en la actualidad 1800 millones de personas consumen agua del grifo contaminada, que hacia 2030 existirá una brecha de $40 \%$ entre la demanda y la oferta de agua potable en los hogares, el $80 \%$ del agua utilizada regresa al entorno sin el tratamiento adecuado, el $30 \%$ de la extracción global de agua se pierde por fugas; desde 1960 ha existido una caída de $55 \%$ en agua dulce disponible per cápita y la escasez de agua afecta actualmente a más del $40 \%$ de la población mundial.

Uhlenbroock (2019) indica que:

La demanda mundial de agua se espera que siga aumentando a un ritmo parecido hasta 2050, lo que representa un incremento del $20 \%$ al $30 \%$ por encima del nivel actual de uso del agua, debido principalmente al aumento de la demanda en los sectores industrial y doméstico. Más de 2.000 millones de personas viven en países que sufren una fuerte escasez de agua, y aproximadamente 4.000 millones de personas padecen una grave escasez durante al menos un mes al año. Los niveles de escasez del agua seguirán aumentando a medida que crezca la demanda de agua y se intensifiquen los efectos del cambio climático. (p.1)

Bakker (2014) explica que en las décadas pasadas se evidenció un crecimiento en el desarrollo de los mercados y la incursión en negocios de gestión de recursos y de gobernanza ambiental involucrando sectores como el del agua, la agroforestería, los humedales, etc. El caso del agua potable remite a que, durante las pasadas dos décadas, en los mercados del agua, se han incluido la privatización de las compañías de suministros de agua, la corporatización de estas compañías, el costeo total del agua en la facturación, el agua embotellada, además de la controvertida privatización de los servicios de agua en los sectores urbanos, tanto que la mayor empresa privada de agua posee 100 millones de usuarios.

Estos hechos demuestran la acogida de la filosofía del ambientalismo del mercado, entendido por Bakker (2014) como "una doctrina fundada en las sinergias entre la protección y conservación ambiental, el crecimiento económico, las economías de mercado y la gobernanza neoliberal" (p.7). Esta posición es controversial, porque en un bucle de realimentación entre lo económico y la protección ambiental, existiendo fallas entre los Estados y los mercados, se vuelve complejo conciliar los intereses de lucro económico con los requisitos de la sostenibilidad ambiental, resolver la tensión entre derechos ambientales y humanos, enfrentados con aquellos de interés para el estado y el mercado; así mismo es complejo encontrar un rol para la comunidad en relación con la gestión del agua.

McNabb (2019) señala que, desde 2018, se ha entrado en una crisis permanente en cuanto a la gestión del agua porque los gerentes deben encontrar, tratar, entregar agua potable desde recursos limitados y en decrecimiento a una gran masa de población que crece rápidamente. Así, existen retos no solo de reemplazo y mantenimiento de infraestructura, lo que significa importantes inversiones, sino también en el aseguramiento que el recurso agua estará disponible en el futuro, un asunto de sostenibilidad; este ítem implica considerar las barreras de tipo climático, de crecimiento demográfico, de urbanización, uso ineficaz del agua en la agricultura, empleo del agua para la generación eléctrica, las fallas en la infraestructura para la entrega del agua.

En este mismo contexto, se requiere sostenibilidad en las ciudades, producción inteligente de energías alternativas, fuentes alternativas de suministro de agua, agricultura sostenible, gestión innovadora del agua, todo dentro de unas metas orientadas a un desarrollo sostenible y por tanto, desde una gobernanza empresarial y del agua con este enfoque de sostenibilidad, pero también de creación de valor empresarial, ambiental y social. 
La representación gráfica de estas ideas relevantes sobre la gobernanza del agua, se desarrolla en el siguiente mapa mental, (Figura 1); que enfatiza en primer término la importancia del agua para la vida y la necesidad de una gobernanza multinivel, bajo la importancia que la gobernanza del agua sea eficiente basado en un conjunto de herramientas de topologías con referencia a las partes interesadas involucradas en enfoques más creativos, con la finalidad de llevar la implementación de las estrategias, indicadores y de la creación de valor comunitario, hacia un aseguramiento de los servicios de agua en el futuro con sustentabilidad y en el marco de la ciudad inteligente, en esencia, es una nueva forma de pensar en localidad con términos de ecoeficiencia, de gestión en las energías limpias y de los hábitats urbanos en función del ser humano, con la idea que la tecnología a través de la Internet de las cosas (IOT), de las redes y sensores inteligentes, y de las comunicaciones móviles, pueden hacer realidad que la ciudad sea vista como un ser vivo con metabolismo propio, insertado dentro de un marco ecosistematico, donde se involucra en relaciones simbióticas y constructivas con el medio que la rodea.

Figura 1

Mapa mental sobre la gobernanza del agua

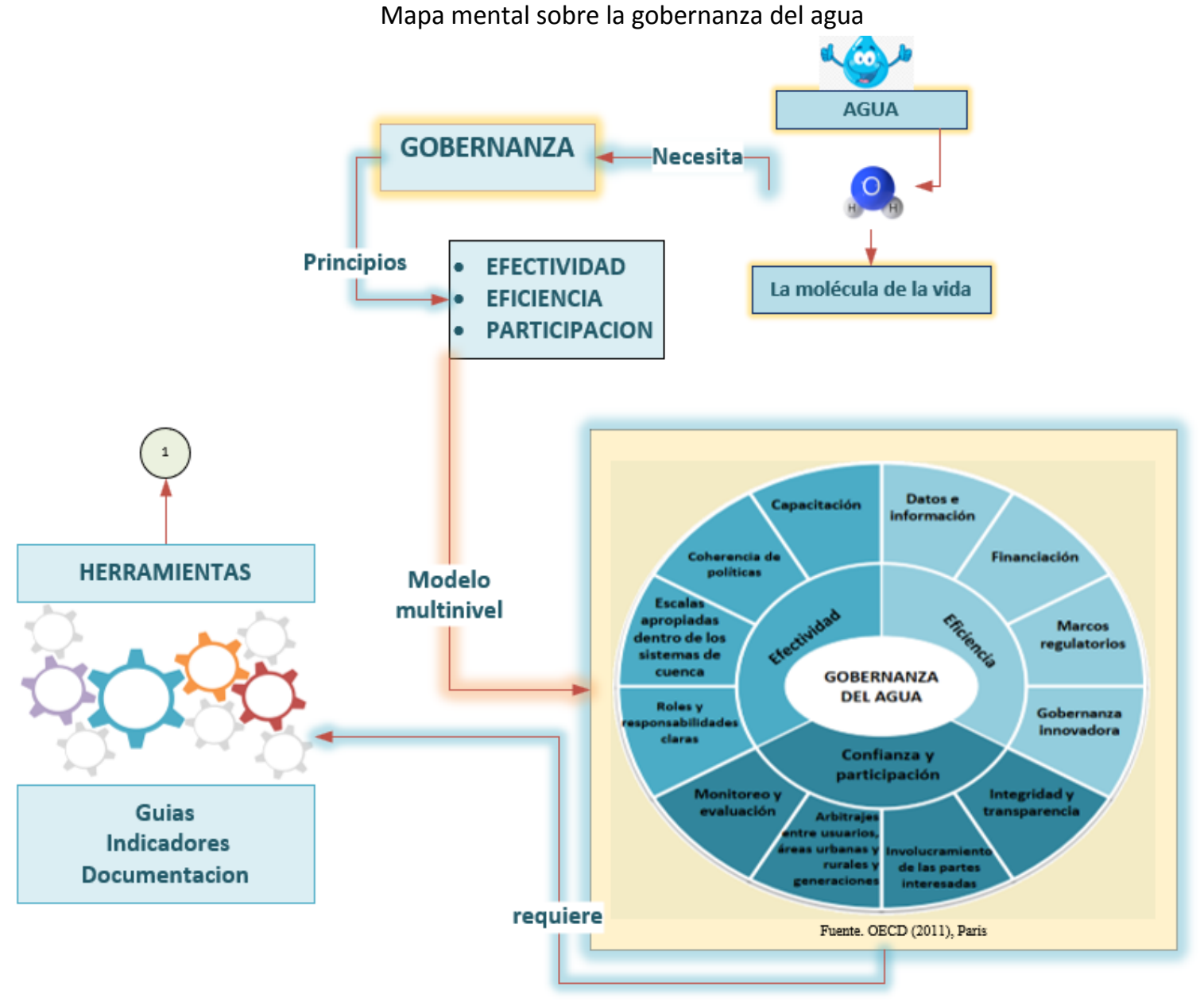




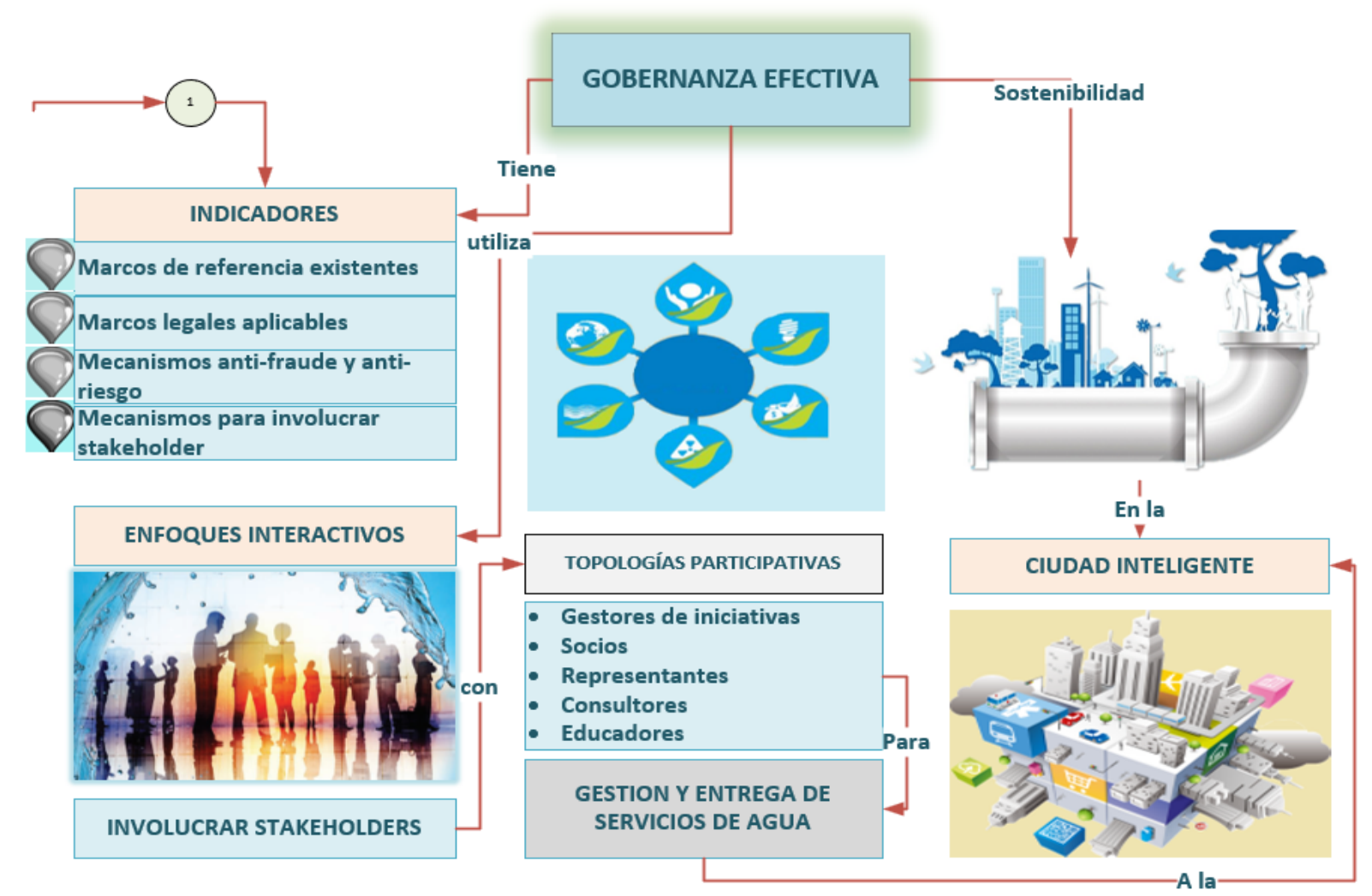

Nota. Modelo de gobernanza multinivel basado en la OECD, que asegure la sustentabilidad en las ciudades inteligentes.

Fuente: Elaboración Pro

Luego, Karar (2017) plantea que la gobernanza del agua en la actualidad, para alcanzar las metas del desarrollo sostenible y en consonancia con las necesidades planteadas por la crisis del agua y la creciente presión sobre los recursos hídricos por la acción antrópica, requiere la migración desde los enfoques tecnocéntrico y ecocéntrico o de tipo híbrido, hacia aquellos de tipo centrado en las personas.

Peña y Solanes (2003) enfatizan en la afirmación que "la crisis del agua es a menudo una crisis de gobernabilidad", línea de acción presentada en el II foro mundial del agua, que tuvo lugar en la Haya, Holanda en 2000 bajo patrocinio de GWP (Global Water Partnership), hecho que puso en el escenario la necesidad de dar prioridad a una gobernabilidad eficaz del agua, teniendo en cuenta, en la gestión de los recursos hídricos, la participación del público y los intereses de todos los colaboradores.

En ese mismo contexto Peña et al. (2003), consignan que "el concepto de gobernabilidad aplicado al agua se refiere a la capacidad social de movilizar energías en forma coherente para el desarrollo sustentable de los recursos hídricos" (p.3). Esta definición de gobernanza del agua lleva implícitas ideas como la manera de consenso social sobre la relación entre agua y sociedad, sobre las políticas públicas que soportan esta relación y la manera en que al aplicar dichas políticas se obtiene la sustentabilidad del agua. Por esto, gobernabilidad implica la capacidad de generar políticas adecuadas y de llevarlas a la práctica.

Es evidente que, existe actualmente una crisis del agua, que se agudiza cada vez más por el aceleramiento del cambio climático, el continuo crecimiento de las ciudades, las presiones que tiene la explosión demográfica sobre la agricultura, los medios de transporte y la demanda de energía; en síntesis, por la necesidad global de agua potable. 
Luego, el problema es, que se requieren concepciones innovadoras, inclusivas, de gobernanza del agua para lo que se avecina. Porcher \& Saussier $(2018$, p.1) indican que "una buena gestión del agua es esencial para el desarrollo humano, la inclusión social, el bienestar de las personas y la sostenibilidad ambiental". La meta es proporcionar acceso universal al agua con buena calidad, dentro de las restricciones financieras existentes, pero esto implica solucionar el debate sobre "si la gestión de los servicios de agua debe ser pública o privada.

Singh et al. (2019); se enfocan en los retos que la gobernanza del agua enfrenta ante la crisis del agua, los requerimientos de sostenibilidad y desarrollo económico, enfatizando en la necesidad de dotar a las instituciones de instrumentos de gestión que les permitan fijar reglas, prácticas y procesos para la toma de decisiones en el manejo de los recursos y servicios hídricos, teniendo en mente el marco de referencia de la gobernanza del agua dado por la Organización para la Cooperación y Desarrollo Económico (OCDE) en 2018.

En particular, las empresas de acueducto y alcantarillado de Colombia son entidades de economía mixtas o privadas que prestan un servicio público y por tanto están regidas por la Ley 142 de 1994, que es la ley marco que regula este tipo de empresas, si bien el agua es vista como recurso y no como derecho fundamental. Entre ellas se tiene a la empresa Acueducto y Alcantarillado de Popayán SA-ESP., empresa mixta, cuyo objeto social es la prestación de los servicios públicos domiciliarios de acueducto y alcantarillado, que consiste en la distribución urbana y rural a nivel municipal de agua apta para el consumo humano, incluida su conexión y medición, a actividades de soporte y primarias, entre ellas las técnico operativas tales como la captación y conducción de agua cruda, procesamiento, potabilización, almacenamiento y distribución de agua tratada, además de la recolección y transporte de aguas servidas por medio de conductos hasta su disposición final.

Siguiendo estas ideas, el problema se puede formular en los términos siguientes: ¿Qué estrategias integradoras de la gobernanza del agua y la creación de valor social en la Sociedad Acueducto y Alcantarillado de Popayán SAESP., conducen a un negocio del agua sostenible?

De esta manera, la investigación se justifica porque se requiere alinear las estrategias de las empresas de acueducto y alcantarillado del país con las tendencias sobre la adopción de modelos de gobernanza del agua multinivel que permitan la participación de las comunidades, tanto en los entornos rurales como urbanos, a fin de paliar la crisis del agua, hacer una mejor gestión de los recursos hídricos, incorporar estrategias integradas de valor social, responsabilidad social empresarial, iniciativas de no marketing, con el cuidado de las cuencas hídricas, el mejoramiento de las condiciones de vida de las personas que realizan cuidado estas en el sector rural o el voluntariado que cuidan los sitios estratégicos de vertimiento de aguas servidas en los entornos urbanos.

Entonces, el objetivo fundamental de la investigación fue diseñar una propuesta con estrategias integradoras de la gobernanza del agua y creación de valor social en el Acueducto y Alcantarillado de Popayán SA-ESP; esto se pudo lograr con los siguientes objetivos específicos: Diagnosticar como se están manejando los procesos de gobernanza del agua al interior del Acueducto y Alcantarillado de Popayán SA-ESP; examinar los elementos potenciales que ofrecen los distintos enfoques teórico-práctico de la gobernanza del agua y la toma de decisiones aplicables al análisis de la gestión del recurso hídrico en el Acueducto y Alcantarillado de Popayán SA-ESP y por ultimo, plantear la propuesta de mejora, identificando las iniciativas potenciales para el fortalecimiento de la gobernanza del agua y los factores que inciden en los procesos de toma decisiones en la gestión del recurso hídrico en el Acueducto y Alcantarillado de Popayán SA-ESP. 


\section{Metodología}

Este es un proyecto de investigación de tipo mixto, de diseño descriptivo, con enfoque disciplinar basado en el modo 2 de producción de conocimiento, apropiado para la gestión de conocimiento del paradigma tecnocientífico.

En la parte cuantitativa se utilizò la encuesta, aplicada a los usuarios de la empresa, cuyo cuestionario se diseñó y aplicó utilizando la herramienta Google Forms; el cuestionario de encuesta constó de 13 preguntas sobre gobernanza del agua, ahorro y uso eficiente del agua.

La población consta de 85.451 usuarios residenciales de la empresa de Acueducto y Alcantarillado de Popayán SA-ESP., distribuidos por estrato, como se muestra en la tabla 1.

\section{Tabla 1}

Distribución de usuarios

residenciales por estrato.

\begin{tabular}{cccc}
\hline Estrato & Usuario Acueducto & Proporción & $\begin{array}{c}\text { Numero de } \\
\text { encuestas }\end{array}$ \\
\hline 1 & 17,391 & 0.203520146 & 22 \\
2 & 26,532 & 0.310493733 & 33 \\
3 & 21,013 & 0.245907011 & 26 \\
4 & 15,906 & 0.186141765 & 20 \\
5 & 4,024 & 0.047091315 & 5 \\
6 & 585 & 0.006846029 & 1 \\
\hline Total & 85,451 & 1 & 106 \\
\hline
\end{tabular}

Fuente. Acueducto y Alcantarillado de Popayán SA-ESP.

La muestra se calcula utilizando la fórmula para poblaciones finitas con igual probabilidad, con un intervalo de confianza del $96 \%$ y un error de $10 \%$, como se muestra a continuación:

$$
n=\frac{\mathrm{N} * \mathrm{p} * \mathrm{q} * z^{2}}{(\mathrm{~N}-1) * e^{2}+p * q * z^{2}}
$$

Donde $\mathrm{N}$ es el tamaño de la población; $\mathrm{p}$, q son las probabilidades de elegir un elemento o no; $\mathrm{z}$ es el valor crítico para un nivel de confianza de $4 \%$, es decir 2.06; e representa el error y $n$ es el tamaño de la muestra.

Utilizando la herramienta en línea de calculadora de muestras del sitio www.aemresearch.com, se obtuvo un tamaño de muestra de 106 encuestas a aplicar.

En cuanto a la parte cualitativa, se seleccionó una muestra de 9 personas, de 190 posibles, utilizando muestreo no probabilístico o por conveniencia, debido a que se estaba interesado en analizar la percepción de las partes interesadas internas de la Sociedad Acueducto y Alcantarillado de Popayán SA-ESP., con referencia a la gobernanza del agua. Como criterio de selección se utilizó personal de dirección, manejo y confianza o personal que tuviera injerencia en los procesos de toma de decisiones administrativas como subgerentes, jefes de divisiones y asistencias. La muestra así seleccionada es la siguiente:

1. Subgerente de Planeación y Estudios

2. Subgerente Administrativo y Financiero

3. Asistencia Subgerencia Técnica y Operativa, acueductos veredales

4. Jefe División Ambiental

5. Jefe División Producción 
6. Jefe División Acueducto

7. Jefe División Alcantarillado

8. Jefe Medición y Control de pérdidas

9. Jefe División Atención Integral al Usuario

De esta manera, se aplicó el cuestionario de entrevista a los anteriores colaboradores, a fin de explorar la concepción que tienen de la gobernanza el agua dentro de la empresa.

De igual forma, para el Focus Group, que es un debate dirigido sobre la temática de la gobernanza del agua, se seleccionó por conveniencia a 8 invitados de los anteriores.

El plan de acción de la investigación se consigna en la figura 2, como se ve a continuación:

Figura 2

Plan de acción

\begin{tabular}{|c|c|c|}
\hline Objetivo & Estrategia & Acciones \\
\hline $\begin{array}{l}\text { Diseñar una propuesta con estrategias } \\
\text { integradoras de la gobernanza del Agsua y } \\
\text { Creación de Valor Empresarial en el } \\
\text { Acueducto y Alcantarillado de Popayan SA- } \\
\text { ESP. }\end{array}$ & ${ }^{8}$ Revisión sistemática de literatura. & $\begin{array}{l}\text { "Explorar libros y articulos recientes sobre } \\
\text { Gobemanza del agua, RSE, marketing } \\
\text { estratégico y valor social. }\end{array}$ \\
\hline $\begin{array}{l}\text { Diagnosticar como se estan manejando los } \\
\text { procesos de gobernanza del agua al interior } \\
\text { del Acueducto y Alcantarillado de Popayán } \\
\text { SA-ESP. }\end{array}$ & $\begin{array}{l}\text { Diseño y aplicación de un cuestionario de entrevista } \\
\text { a funcionarios administrativos y operativos } \\
\text { involucrados proceso de gobermanza del agua dentro } \\
\text { del Acueducto y Alcantarillado de Popayan SA-ESP. }\end{array}$ & $\begin{array}{l}\text { "Revisar en la literatura los conceptos de } \\
\text { gobermanza y gobernanza del agua. } \\
\text { "Revisar la literatura sobre sostenibilidad, } \\
\text { creación de valor social y empresarial. } \\
\text { "Identificar estrategias para la gobermanza } \\
\text { del agua y la RSE. } \\
\text { "Diseñar cuestionario de entrevista en } \\
\text { concordancia con las revisiones realizadas. }\end{array}$ \\
\hline $\begin{array}{l}\text { Examinar los elementos potenciales que } \\
\text { ofrecen los distintos enfoques teórico- } \\
\text { prácticos de la gobernanza del agua y la toma } \\
\text { de decisiones aplicables al analisis de la } \\
\text { gestión del recurso hidrico en el Acueducto y } \\
\text { Alcantarillado de Popayán SA-ESP. }\end{array}$ & $\begin{array}{l}{ }^{8} \text { Explorar los diferentes modelos de gobermanza del } \\
\text { agua. } \\
\text { "Explorar la creación de valor social en la parte baja } \\
\text { de la pirámide. } \\
\text { "Explorar la percepción sobre gobernanza del agua } \\
\text { de los usuarios extemos de la Sociedad Acueducto y } \\
\text { Alcantarillado de Popayán SA-ESP., a través de una } \\
\text { encuesta. }\end{array}$ & $\begin{array}{l}\text { *Crear mapas conceptuales con Cmap tools. } \\
{ }^{*} \text { Crear mentefactos con Visio. } \\
\text { *Analizar y difundir los resultados de la } \\
\text { encuesta. } \\
\text { *Crear un blog para comunicar los diferentes } \\
\text { aspectos de la gobernanza del agua. }\end{array}$ \\
\hline $\begin{array}{l}\text { Plantear la propuesta de mejora, } \\
\text { identificando las iniciativas potenciales para } \\
\text { el fortalecimiento de la gobemanza del agua } \\
\text { y los factores que inciden en los procesos de } \\
\text { toma decisiones en la gestión del recurso } \\
\text { hidrico en la Sociedad Acueducto y } \\
\text { Alcantarillado de Popayan SA-ESP. }\end{array}$ & $\begin{array}{l}\text { "Diseñar e implementar un focus group para la } \\
\text { concientización sobre la crisis del agua, el derecho } \\
\text { fundamental al agua y la responsabilidad del } \\
\text { ciudadano y usuario en la conservación del agua, asi } \\
\text { como los retos a la Sociedad Acueducto y } \\
\text { Alcantarillado de Popayán SA-ESP., en lo } \\
\text { relacionado con la gobernanza del agua. } \\
\text { "Incluir en el blog corporativo de gobemanza del } \\
\text { agua un Web Mix con enlaces de interés, videos y el } \\
\text { voluntariado comunitario. }\end{array}$ & $\begin{array}{l}\text { * Discusión de los factores e impactos de la } \\
\text { crisis del agua en el escenario mundial y } \\
\text { colombiano. } \\
\text { "Discusión del derecho fundamental del } \\
\text { agua, la comunidad y su participación en el } \\
\text { cuidado del liquido vital. } \\
\text { " Promover el voluntariado sobre cuidado } \\
\text { del agua como recurso vital y derecho } \\
\text { fundamental. } \\
\text { "Difundir la propuesta y los resultados del } \\
\text { focus group en el blog creado. }\end{array}$ \\
\hline
\end{tabular}

Nota. Propuesta con estrategias y acciones integradoras de la gobernanza del agua y creación de valor social en la empresa.

Fuente: Elaboración propia.

\section{Resultados y discusión}

Los resultados de la encuesta a los usuarios residenciales de la Sociedad Acueducto y Alcantarillado de Popayán SA-ESP., bajo investigación revelan que:

1. El $63.1 \%$ saben que el agua es un recurso limitado, del cual depende la vida misma.

2. Existe un consenso mayoritario, que el agua es un derecho fundamental del ser humano (98.1\%) y que conlleva corresponsabilidad para cuidar de este recurso limitado. 
3. El $87.6 \%$ de los usuarios encuestados, afirman que gradúan el grifo de sus lavaderos cuando consumen agua en sus labores domésticas, lo que implica que perciben el valor del agua mucho más allá de los impactos económicos que el desperdicio puede conllevarles.

4. Con referencia a la reutilización de las aguas grises en los servicios sanitarios y de las aguas lluvias para este mismo propósito u otros de uso del hogar como lavar pisos o regar las plantas, solamente $57.1 \%$ de los encuestados.

5. El $75.2 \%$ de los usuarios encuestados desconozcan el marco legal que habla del mínimo vital, particularmente cuando la mayoría son de los estratos favorecidos, es decir 1 y 2 , y siendo que este mecanismo trata de cumplir parcialmente el mandato de las Naciones Unidas sobre el agua como derecho fundamental. Se requiere aplicación y difusión de estos beneficios.

6. En cuanto a la protección de las fuentes de agua, dado que las aguas grises van a las fuentes hídricas, la acción preventiva debe iniciar en el hogar y por ello los desperdicios sólidos de lavaderos o lavamanos deben filtrarse mediante rejillas, las cuales son usadas por $95.2 \%$ de los usuarios encuestados.

7. En cuanto a la agudización de la crisis del agua, el $98.1 \%$ de los encuestados afirman que en este contexto el agua deberá racionarse.

8. El 94.3\% de los usuarios encuestados del AAP SA-ESP., está dispuesto a emprender acciones para cuidar el agua y evitar el desperdicio.

9. Dentro de las acciones de gobernanza del agua comunitarias, especialmente en los orígenes de las fuentes hídricas, o en las acciones encaminadas a protegerlas, es bien visto el pago por servicios ambientales a quienes trabajen en estas labores; esto lo refleja el hecho que $82.5 \%$ de los encuestados del AAP SA-ESP., esté de acuerdo con esta medida pecuniaria.

10. El $87.6 \%$ de los usuarios encuestados del AAP SA-ESP., está dispuesto a unirse en voluntariado a las acciones involucradas en la gobernanza del agua, en aras de la conservación del agua sobre la faz del planeta tierra.

11. En cuanto a la calidad del agua que el Acueducto y Alcantarillado de Popayán SA-ESP., les ofrece, afirma el $86.7 \%$ de los encuestados que es buena.

Los resultados de la entrevista a los funcionarios de las áreas y secciones relacionadas con la gobernanza del agua y la toma de decisiones concomitantes en la empresa de acueducto y alcantarillado en estudio, se sintetizan así:

Con referencia a la categoría gobernanza, gobernanza del agua y gobernanza corporativa, los entrevistados manifestaron que: La gobernanza es vista como las diferentes interrelaciones de los componentes de la sociedad en un marco de sociabilidad, bajo supuesto que todos aceptan sus responsabilidades y participan en las relaciones que buscan construir políticas, estrategias, desde las personas, la gente, de abajo hacia arriba, con la finalidad de mejorar los gobiernos, la gobernabilidad, involucrando las ramas judicial, legislativa y ejecutiva, de manera que se construye desde la acción empresarial, traduciéndose esta acción en gobernanza corporativa, una serie de acciones integrales, que van más allá de marketing, enfocadas en la creación de valores agregados para la empresa a través de la responsabilidad social empresarial que, en caso de las empresas de acueducto y alcantarillado, tienen relación con la conservación de las fuentes hídricas y la prestación de un servicio continuo y con calidad, a la comunidad, a la que involucran de forma activa, deviniendo entonces en gobernanza del agua, buscando en particular, que se preserven las cuencas en las partes altas, con la ayuda de las comunidades y con el objetivo principal de mantener la calidad del agua en su origen.

Luego, la gobernanza del agua se puede ver las acciones coordinadas, para la conservación, gestión y aseguramiento del recurso finito agua, con la participación de todas las partes interesadas, teniendo criterios de sustentabilidad y creación de valor social, que tiene como componente muy importante, la responsabilidad social empresarial, desde una perspectiva amplia, integral y obligatoria, con componentes no marketing, dirigidos hacia la base de la pirámide. 
Con referencia a la categoría sostenibilidad, las entrevistas revelan que: La sostenibilidad, ésta se mira desde la gestión hídrica particularmente de las cuencas en su parte superior, donde las acciones de conservación se enmarcan dentro de la gobernanza ambiental, donde se ha buscado la cooperación con las comunidades indígenas y campesinas, a la vez que se han buscado los recursos con organizaciones no gubernamentales como el Banco Interamericano de Desarrollo; también se han involucrado los esfuerzos en la gestación de plantas de tratamiento de aguas residuales a nivel urbano. Se trata no solamente minimizar los impactos negativos a las fuentes hídricas, sino también de asegurar el recurso para prestar el servicio de forma continua, con calidad y de llegar a más usuarios, es decir, de ampliar la cobertura; también de ampliar las redes de distribución para lo cual se involucrado la mano de obra de las comunidades, haciendo acción social en la base de la pirámide.

A la sostenibilidad contribuye también los proyectos de nuevas plantas de tratamiento como las de río Palacè, donde se planea tener una capacidad de entrega con un caudal de 500 litros por segundo, y es un recurso valioso para el futuro, hacia 2050, cuando la prospectiva indica que se va a doblar la demanda de agua en la ciudad de Popayán, lo que presupone no solamente trabajar por mantener los niveles de sedimentación bajos, conservar el cauce y obviamente el recurso hídrico; las acciones de concientización de la comunidad con referencia a que utilicen los recursos hídricos locales, existentes fundamentalmente en el medio rural, como son los nacimientos de agua, los aljibes, para que con esta agua realicen labores que no implican una alta potabilidad del agua, como acciones de regadío, de ganadería, de aseo personal, y que con el agua tratada que les llega, cocinen los alimentos.

Con respecto a la creación de valor social, las entrevistas ponen en evidencia que: La comunidad es importante cuando se trata de diagnóstico de las necesidades en cuanto a la infraestructura de la distribución de agua, como construcción de plantas de tratamiento de aguas residuales, o cuando en el sector rural, en los ámbitos campesinos e indígenas se hace un manejo conjunto del cuidado de las cuencas, pero es a las empresas de acueducto y alcantarillado que les corresponde la gestión de recursos, mediante generación de proyectos, en el orden nacional, pero falta más acciones de comunicación a la comunidad, más educación sobre la necesidad de racionalizar el consumo del agua, particularmente en los entornos urbanos, donde esta requiere prioridad la potabilización.

Por tanto, la interrelación con las comunidades para proteger el recurso limitado del agua, está encaminado no solamente a su conservación sino también a abaratar los costos de potabilización, y mantener una alta calidad del producto con lo cual se benefician las comunidades, los usuarios; a nivel de la gobernanza corporativa, se tratan de obtener una alta eficiencia en la coordinación de las acciones que permitan la conservación, la distribución, el control, la calidad del agua; son acciones de gestión y de hecho, la Sociedad Acueducto y Alcantarillado de Popayán SA-ESP.

En cuanto a las estrategias de gobernanza del agua, los entrevistados manifiestan que: Los usuarios del Acueducto y Alcantarillado de Popayán SA-ESP., pagan sus tarifas porque reconocen la alta calidad del agua ofrecida por la institución y los esfuerzos de esta para mantenerla y para aumentar la cobertura; además, la construcción de la imagen empresarial y su percepción positiva por parte de los usuarios, las tecnologías de la información y la comunicación, a través de la página web institucional, donde se presta especial interés a los procesos de atención integral al usuario, en particular a la gestión de las peticiones, quejas, reclamos y solicitudes de nuevos servicios.

Esto ha sido posible debido al impulso que se le está dando dentro de la organización al empleo de tecnologías digitales como por ejemplo el programa compás proporcionado por el gobierno Suizo o la utilización del software Aquarisk, como pilares fundamentales de la transformación tecnológica dentro de la Sociedad Acueducto y Alcantarillado de la ciudad; esto podría ser considerado como ciertos rasgos de innovación de la gestión de la 
información dentro de la empresa, pero no la gobernanza del agua, porque de hecho, está como tal no existe en la institución.

Los resultados del focus group pueden sintetizarse así: En el dominio rural particularmente existen avances en aspectos de la política nacional de gestión del recurso hídrico, por que se construye con la comunidad un modelo propio de gestión; donde existe un marco de respeto y una visión compartida que el agua es vida, con construcción de diálogo de saberes que permitan establecer acuerdos para paliar la crisis del agua y para empezar a crear acciones de gobernanza del agua, que involucren a las partes interesadas.

Así, los procesos de gobernanza corporativa deben estar enlazados con los principios de concertación, participación y construcción de un bienestar social, de modo que, se proyecten hacia afuera para incluir las comunidades en la conservación de las cuencas hídricas, en el ahorro y uso eficiente del agua, en la minimización de la contaminación del agua, aguas abajo, en la creación de conciencia de la necesidad que el agua debe utilizarse de forma eficiente y hay que ahorrar porque es un recurso limitado.

Dentro del grupo de enfoque se enfatiza que, para lograr paliar la crisis del agua, en un escenario mundial sometido al cambio climático y a las presiones demográficas aceleradas, es necesario involucrar a las comunidades dentro de un modelo de gobernanza del agua para respetar las diferentes cosmovisiones que se tienen con referencia al agua y al territorio, fomentar la eficiencia productiva de las comunidades, darles mejores niveles de calidad de vida, darle lugar a la equidad social, para que puedan dedicar parte de sus actividades diarias a las labores de conservación, como por ejemplo el pago por servicios ambientales que es una herramienta que reconoce estos esfuerzos a las comunidades, particularmente campesinas e indígenas.

Se propone un modelo multinivel de gobernanza del agua, donde el ahorro y el uso eficiente del agua por parte de los usuarios, los procesos de reciclaje para una baja contaminación, sustentables, la aplicación de la tecnología no sólo para tratar el agua sino también para soportar la manera de vivir equilibrada dentro de la ciudad inteligente del futuro, son fundamentales para que tenga éxito y se pueda disponer de reservas de agua de forma sustentable para el futuro.

De igual forma, se resalta la importancia que se le da a la aplicación de las tecnologías de la información y a los avances científicos con referencia al tratamiento del agua, las estrategias de agricultura biológica, de Fitorremediación, de utilización de fuentes alternativas de energía, de diseño de sistemas ecológicos, de avances en inteligencia artificial y nanotecnología, que soportan la evolución hacia la Internet de las cosas, la consolidación de una ciudad vista como un sistema vivo, con metabolismo propio, que se conceptualiza como ciudad inteligente, en la que la sustentabilidad es una de las metas y la gobernanza del agua es una de sus necesidades porque persigue no sólo la conservación de la naturaleza, sino también el reciclaje, la reutilización, la no contaminación, el uso eficiente, de los recursos escasos, como es el agua.

Los participantes del Focus Group concluyen que, en primer término, la crisis del agua es una crisis de la gobernanza del agua, porque se tiene que cambiar la manera en que se gestiona el agua, aprovechando los recursos para gestionar de forma más eficiente y preservar las cuencas hídricas, pactando con entidades internacionales que hayan tenido experiencias exitosas en gestión y preservación de las fuentes de agua, involucrando, dentro de un marco de respeto y concertación, a las comunidades indígenas y campesinas, que desde su quehacer, contribuyen de forma definitiva la gobernanza del agua.

Enfatizan también, que se debe buscar un equilibrio entre los intereses de las distintas partes interesadas, teniendo en cuenta que en el mundo la tendencia es hacia la privatización y la cogestión de recursos fronterizos de agua, con el fin de obtener mejor gobernanza de este recurso y paliar la crisis existente; por ello, desde la academia es pertinente promover la investigación sobre los modelos de gobernanza del agua para poder crear 
cultura del agua y avanzar en este tópico, de manera que dentro de las organizaciones de acueducto y alcantarillado, se promuevan capacitaciones y se creen secciones dedicadas a la gobernanza del agua.

En este orden de ideas, los participantes expresaron su deseo que, experiencias como las de este Focus Group se institucionalicen y se vuelvan más inclusivas e internacionales de ser posible, puesto que el agua lo necesitamos todos los seres vivos.

De hecho, existe corresponsabilidad con referencia a la gobernanza del agua y esto implica que los usuarios, cuando son residenciales, la utilicen de forma eficiente, no la desperdicien, reciclen aguas grises, coloquen rejillas, utilicen aguas lluvias para las labores no vitales dentro de sus hogares.

Esto también lleva, a considerar, pagos por servicios de cuidado de las aguas que van a ser utilizadas aguas abajo, es decir fomentar una cadena de cuidado de las fuentes hídricas, porque quienes estamos arriba, de los cauces, si contaminamos, no solamente afectamos nuestro entorno local sino también a aquellos que están, en las partes bajas.

\subsection{Discusión de resultados}

Los propósitos planteados en el plan de acción y cumplidos a través de los instrumentos, entre otros, desembocan a un análisis de resultados sobre tendencias de gobernanza del agua en el mundo en 2020, desde autores como (UNESCO, 2020); (Naciones Unidas, 2020); (Huang et al., 2020); (Te Wierik et al., 2020); (Wilson, 2020); (Pinos, 2020); (Schreiner \& van Koppen, 2020); (Pahl-Wostl et al., 2020); (OECD, 2018); (IWRA, 2020); (McDonald, 2020); (Jiménez et al., 2020), encontrándose coincidencia absoluta entre todas las inquietudes y tendencias planteadas por ellos con referencia a la gobernanza del agua en el mundo, particularmente en Latinoamérica, y los resultados hallados por esta investigación, de forma que, se apunta a la necesidad de introducir dentro de las empresas de acueducto y alcantarillado un modelo de gobernanza multinivel del agua, basado en los principios, marco metodológico de implementación de indicadores propuestos por la OCDE, teniendo en cuenta los lineamientos generales de nivel internacional y las particularidades de las partes interesadas a nivel local y regional.

La propuesta consiste en que la Sociedad Acueducto y Alcantarillado de Popayán SA-ESP, adopte como modelo de gobernanza del agua la estrategia multinivel con los principios, indicadores y marco metodológico de evaluación, propuestos por la OCDE, pero enfatizando en las particularidades locales de sus micro entornos y partes interesadas. Esto implica, que se incluyan los siguientes elementos dentro de este modelo de gobernanza multinivel del agua:

1. Institucionalizar la realización de foros de discusión, Focus Group y encuentros locales, regionales, nacionales y de orden internacional sobre la gobernanza del agua.

2. Promover el derecho fundamental al agua y el mínimo vital, teniendo en cuenta que el agua fluye, es dinámica, está en constante cambio, donde este vital liquido es de todos, para todos y conecta a todo, además es responsabilidad de cada ser humano contribuir a su conservación, su uso eficiente, su estado de no contaminación; por tanto, quienes están aguas arriba deben pensar en los que están aguas abajo.

3. Incorporar la innovación tecnológica en la medición y control del agua servida, así como también, los procesos de tratamiento y potabilización, con miras a una gobernanza del agua en la ciudad inteligente.

4. Fortalecer la capacitación del talento humano de la Sociedad AAP SA-ESP., sobre gobernanza del agua y la comunicación de experiencias, proyectos y servicios relacionados con la gobernanza multinivel, por ejemplo, acciones de gestión del agua adelantadas por la fundación Procuenca Río las Piedras (senderos ecológicos, agricultura sostenible, reforestación de cuencas hídricas), experiencias de cuidado de cuencas, construcción 
y mantenimiento de redes que involucren a las comunidades indígenas y campesinas o a usuarios de estratos vulnerables.

5. Incorporar dentro de la agenda de la gobernanza multinivel del agua procesos que devengan en programas de cultura del uso y ahorro eficiente del agua, de pago de servicios ambientales, o de tasas para quienes efectúen mayores desperdicios del agua o la contaminen drásticamente; también recibir transferencias de recursos, de entidades del sector del agua de orden local, regional, nacional y del exterior, cuando se demuestra que aguas abajo, los flujos de estas es menos contaminada y de mejor calidad, recursos que se invierten en acciones de minimización de impactos, de conservación de cuencas y de la calidad del agua, y también para financiar aquellos que están aguas arriba haciendo una acción similar.

6. Establecer procesos de prospectiva estratégica para la evaluación de escenarios posibles de mediano y largo plazo sobre la condición de las cuencas hídricas que abastecen la ciudad de Popayán, utilizando herramientas software que utilicen algoritmos desde la inteligencia artificial.

7. Dar a conocer las acciones realizadas sobre la gobernanza multinivel del agua a través de la página web corporativa, y herramientas Web 2.0 como blogs, infografías, videos, mapas mentales o conceptuales, que contribuyan a la creación de conciencia sobre la necesidad de uso eficiente de un recurso limitado como es el agua, entre todos los ciudadanos.

8. Establecer mecanismos para que se agilice la construcción de la planta de tratamiento de aguas residuales en el sector suroccidental de la ciudad de Popayán, con el fin de descontaminar las microcuencas de los ríos Molino y ejido, entre otras, que vierten sobre la cuenca principal del río Cauca, minimizando impactos ambientales en gran medida porque por esta obra civil pueden pasar gran parte de las aguas grises o servidas de la ciudad mejorando las condiciones de vida de los usuarios que habitan estas microcuencas.

9. En las áreas urbanas, con el fin de cuidar las cuencas hídricas, promover acciones para evitar la contaminación con desechos de construcciones, pinturas y sus derivados, materiales no biodegradables y electrónicos entre otros, se debe instaurar el voluntariado comunitario, consistente en que se involucran usuarios para el cuidado y vigilancia de puntos clave de estas cuencas, remunerándolos con pagos ambientales vía tarifas en los servicios prestados por la Sociedad Acueducto y Alcantarillado de Popayán SAESP.

\subsection{Estudios Futuros}

En primer término, es necesario establecer de forma completa el costo del cumplimiento de las metas involucradas dentro del SDG6, las metas deben desarrollo global sostenible referente a la gobernanza del agua en el mundo porque como señalan (Guppy \& Anderson, 2017a) solamente cumplir con las metas de suministro de agua, salubridad e higiene cuesta 114. mil millones de dólares anuales, sin tener en cuenta los costos de mitigación o recuperación de los sistemas hídricos, la degradación de los ecosistemas, los daños a la infraestructura por las inundaciones, efectos del desequilibrio acelerado del clima mundial causado por el ser humano, tienen sobre la economía, además que por estos efectos ya mueren en el mundo 12.6 millones de personas. Esto es la crisis del agua que están allí a la vista de todos y que, desafortunadamente, no se tiene plena conciencia de ella.

(Dadson et al. 2020) exponen como tendencias de importancia para paliar la crisis del agua, y por tanto, obtener un mejoramiento de la gobernanza del agua en el futuro, temáticas, dentro de las ciencias hidrológicas, como la determinación de los extremos hidro climáticos en un mundo con cambio climático, investigaciones sobre los sistemas hídricos subterráneos, la creación de modelos de gestión, vigilancia y control de la calidad del agua, la determinación de los retos que se enfrentan en los sistemas de agua dulce, los retos de saneamiento básico 
higiene con relación al agua y la modelización y vigilancia de los procesos hidrológicos; con referencia a las políticas, se tienen la reubicación del agua, la construcción de políticas hídricas rurales en continentes como Asia y África, las políticas sobre el derecho fundamental al agua, la gestión de las crecientes e inundaciones, el marco de referencia para las políticas sobre cobros por el desperdicio del agua, los planes de acción contra las sequías; en el dominio de la gestión del agua, se proponen en el modelamiento y el análisis de datos para la toma de decisiones con referencia a los sistemas hídricos, financiar la infraestructura del agua, procesar las aguas contaminadas para convertirlas en un recurso útil, actuar sobre los modelos urbanos para asegurar la disponibilidad del agua y a la vez obtener mayor justicia social y sobre todo fortalecer la educación con referencia al agua, a su uso eficiente y a su ahorro.

De hecho, para una mejor gobernanza del agua en el futuro, se necesita también avanzar en la implementación de la ciudad inteligente, de manera que los edificios sustentables o verdes, puedan contar con dispositivos tecnológicos que permitan una ciudad sustentable, donde el Antropoceno, se caracterice por ser sustentable, con justicia social, centrado en los seres humanos y no en economía. Matos, (2020) parte del hecho que el agua es el solvente universal y el componente fundamental para la persistencia de la vida en el planeta que y por ello, propone medidas de gobernanza, es decir de arriba hacia abajo, para luchar contra el cambio climático, desde los espacios públicos de las ciudades, mediante mejores canales, parques, espacios recreativos, que se insertan dentro de un diseño eto-ecológico de la ciudad; esto incluye bio retenedores para la lluvia, pavimentos porosos, recuperación y rehabilitación de corrientes hídricas, cansada sumergibles, entre otros.

De igual forma, (Sheth, 2017) establece pautas tecnológicas para obtener un uso eficiente del agua en los edificios verdes de la ciudad inteligente; tuberías y llaves de bajo flujo, orinales y sanitarios eficientes en el uso del agua, lavadoras y electrodomésticos que utilicen eficientemente el agua, auditorías del agua en todos los edificios, utilización eficiente del agua durante las construcciones e igualmente en las irrigaciones, recolección de aguas incluyendo las lluvias y tratamiento, reutilización, reciclaje de otras aguas, como son las lluvias tomadas grises, aquellas de los servicios sanitarios, sistemas de pavimentación porosa, recubrimiento con capas vegetales de los suelos, entre otros.

Entonces, estas tendencias apuntan hacia los modelos multinivel de la gobernanza del agua y hacia las 3P, expuestas por Romano \& Akhmouch (2019): centralidad de personas, procesos y lugares, de forma que la gobernanza sea más que un medio, un fin que se más que dentro de la ciudad inteligente y sustentable del futuro, e igualmente dentro de un mundo con una economía limpia y en función del ser humano, donde se tienen que concertaba acuerdos de gestión de recursos de agua transfronterizos.

\section{Conclusiones}

De acuerdo a los resultados de esta investigación, se puede afirmar que no existe gobernanza del agua en la Sociedad Acueducto y Alcantarillado de Popayán SA-ESP., pero existen acciones importantes de gestión del agua que incluyen la conservación de cuencas hídricas en las partes altas, con participación de las comunidades indígenas y campesinas

Según esta investigación, se encuentra confusión entre los conceptos de gobernanza, gobernabilidad y gobernanza del agua y por ello se considera que las acciones de gestión del agua son de gobernanza de este líquido vital, y que la participación comunitaria y la gestión del agua, es creación de valor social, cuando en verdad, la gestión del agua es el paso inicial hacia la construcción de un modelo multinivel, sociocéntricos, dinámico, inclusivo y de gobernanza del agua.

La gobernanza es vista como una herramienta de interacción entre instituciones y sociedad, de abajo hacia arriba, para la toma de decisiones que devienen políticas que buscan mejorar la gobernabilidad, que se traduce en las 
instituciones en gobernanza corporativa, vista como las diferentes acciones de las secciones, divisiones, departamentos, de una organización, con miras a la consecución de sus objetivos empresariales.

De acuerdo a los resultados de la investigación, existe conciencia en los usuarios residenciales de la Sociedad Acueducto y Alcantarillado de Popayán SA-ESP, que el agua es un recurso limitado y que debe cuidarse porque es insustituible, porque se puede llegar a una crisis más crítica del agua a nivel local; sin embargo, los usuarios no la usan eficientemente, no utilizan las aguas lluvias, no reciclan las aguas grises, no están conscientes que deben aminorar los impactos de contaminación en las fuentes hídricas, lo que implica la necesidad de fortalecer programas a una nueva cultura del agua y comunicar las experiencias sobre gobernanza de la molécula de la vida.

Dentro de los elementos adicionales adecuados a las necesidades particulares de la ciudad de Popayán y de los intereses de sus partes interesadas con referencia a la gobernanza del agua, están la implementación de pagos por servicios ambientales a las comunidades indígenas y campesinas que cuidan y conservan las cuencas hídricas en las partes altas, la creación de bancos de agua, de manera que en estas comunidades, en épocas de sequía pueda gozar del servicio de agua potable apta para el consumo humano, la creación de la cadena del agua, pagos o cuotas, para quienes aguas arriba cuidan el agua para que se disfrute de mejor calidad de esta aguas abajo, la de tasas que castiguen a quienes excedan los niveles de contaminación de las fuentes hídricas.

\section{Referencias bibliográficas}

Bakker, K. (2014). The Business of Water: Market Environmentalism in the Water Sector. Annual Review of Environment and Resources, 39(1), 469-494. https://doi.org/10.1146/annurev-environ-070312-132730

Dadson, S., Garrick, D., Penning, E., Hall, J., Hope, R., \& Hughes, J. (2020). Ciencias del agua, política y gestión: un reto global. Willey.

Guppy, L., \& Anderson, K. (2017a). Global Water Crises: The facts (p. 17). UNU INWEH.

Guppy, L., \& Anderson, K. (2017b). Global Water Crisis: The Facts. United Nations University.

Huang, C., Chen, W., \& Yi, H. (2020). Collaborative networks and environmental governance performance: a social influence model. Public Management Review, 1-22.

https://doi.org/10.1080/14719037.2020.1795229

IWRA. (2020). OECD Principles on Water Governance. In OECD Principles on Water Governance (Issue 16). Routledge. https://doi.org/10.4324/9780429448058

Jiménez, A., Saikia, P., Giné, R., Avello, P., Leten, J., Lymer, B. L., Schneider, K., \& Ward, R. (2020). Unpacking water governance: A framework for practitioners. Water (Switzerland), 12(3), 1-21.

https://doi.org/10.3390/w12030827

Karar, E. (2017). Freshwater governance in 21st Century (Vol. 6). Springer. https://doi.org/10.1007/978-3-31943350-9

Matos, M. (2020). Ciudades sostenibles: Espacios públicos para el agua. CRC Press.

McDonald, D. A. (2020). Innovation and new public water. Journal of Economic Policy Reform, 23(1), 67-82. https://doi.org/10.1080/17487870.2018.1541411

McNabb, D. E. (2019). Global Pathways to Water Sustainability. In Global Pathways to Water Sustainability. Springer. https://doi.org/10.1007/978-3-030-04085-7

Naciones Unidas. (2020). UN-Agua: estrategia 2030 (p. 36). Naciones Unidas. 
OECD. (2018). OECD Water Governance Indicator Framework (p. 35). OECD. https://doi.org/10.1787/9789264292659-5-en

Pahl-Wostl, C., Knieper, C., Lukat, E., Meergans, F., Schoderer, M., Schütze, N., Schweigatz, D., Dombrowsky, I., Lenschow, A., Stein, U., Thiel, A., Tröltzsch, J., \& Vidaurre, R. (2020). Enhancing the capacity of water governance to deal with complex management challenges: A framework of analysis. Environmental Science and Policy, 107(July 2019), 23-35. https://doi.org/10.1016/j.envsci.2020.02.011

Peña, H., \& Solanes, M. (2003). Gobernabilidad efectiva del agua (p. 44). CEPAL NU. http://marefateadyan.nashriyat.ir/node/150

Pinos, J. (2020). Multiple water governance models: Ecuador as a case study. Maskana, 11(1), 74-80. https://doi.org/10.18537/mskn.11.01.08

Porcher, S., \& Saussier, P. (2018). Facing the Challenges of Water Governance. In Facing the Challenges of Water Governance. Palgrave. https://doi.org/10.1007/978-3-319-98515-2

Romano, O., \& Akhmouch, A. (2019). Water governance in Cities: Current trends and future challenges. Water (Switzerland), 11(3). https://doi.org/10.3390/w11030500

Schreiner, B., \& van Koppen, B. (2020). Hybrid water rights systems for pro-poor water governance in Africa. Water (Switzerland), 12(1), 1-8. https://doi.org/10.3390/w12010155

Sheth, K. N. (2017). Water Efficient Technologies For GreenBuildings. International Journal of Engineering Innovation and Scientific Research, 1(3), 2395-6372.

Singh, A., Saha, D., \& Tyangi, A. (2019). Water Governance : Challenges and Prospects. Springer.

Te Wierik, S. A., Gupta, J., Cammeraat, E. L. H., \& Artzy-Randrup, Y. A. (2020). The need for green and atmospheric water governance. Wiley Interdisciplinary Reviews: Water, 7(2), 1-20.

https://doi.org/10.1002/wat2.1406

Uhlenbroock, S. (2019). Informe Mundial de las Naciones Unidas sobre el Desarrollo de los Recursos Hídricos 2019. No dejar a nadie atrás. In Organización de las Naciones Unidas para la Educación, la Ciencia y la Cultura. UNESCO. http://www.unwater.org/publications/world-water-development-report-2019/

UNESCO. (2020). Informe Mundial de las Naciones Unidas sobre el Desarrollo de los Recursos Hídricos 2020: Agua y Cambio Climático. UNESCO.

Wilson, N. J. (2020). Querying water co-governance: Yukon first nations and water governance in the context of modern land claim agreements. Water Alternatives, 13(1), 93-118.

Esta obra está bajo una Licencia Creative Commons Attribución-NoCommercial 4.0 International

(c) B BY-NC 